\title{
From flapping wings to underactuated fingers and beyond: a broad look to self-adaptive mechanisms
}

\author{
L. Birglen \\ Ecole Polytechnique of Montreal, Department of Mechanical Engineering, \\ Ecole Polytechnique of Montreal, Montreal, QC, H3T 1J4, Canada
}

Received: 7 March 2010 - Revised: 29 June 2010 - Accepted: 19 August 2010 - Published: 22 December 2010

\begin{abstract}
In this paper, the author first reviews the different terminologies used in underactuated grasping and illustrates the current increase of activity on this topic. Then, the (probably) oldest known self-adaptive mechanism is presented and its performance as an underactuated finger is discussed. Its original application, namely a flapping wing, is also shown. Finally, it is proposed that the mechanisms currently used in underactuated grasping have actually other applications similarly to the previously discussed architecture could be used for both an underactuated finger and a flapping wing.
\end{abstract}

This paper was presented at the IFToMM/ASME International Workshop on

Underactuated Grasping (UG2010), 19 August 2010, Montréal, Canada.

\section{Self-adaptive, adaptive, intelligent, underactuated, differential, or compliant?}

Self-adaptive mechanisms as used in underactuated fingers (Birglen et al., 2008) are often confused with other classes of mechanisms because of the lack of a clear definition. According to Gosselin (2006), adaptive mechanical systems are defined as systems "in which the ability to adapt to new external situations relies strictly on mechanical properties" (quoted). Although the denomination of "adaptive mechanisms" proposed in the latter reference is absolutely correct, the author of this paper often prefers referring to them as "self-adaptive mechanisms" to avoid confusion. Indeed, the latter expression emphasizes that the adaptation capability refers to the mechanical system itself and does not describe an algorithmic procedure. As noted in Gosselin (2006), adaptive systems are usually associated with computer systems and this has lead to some confusion in underactuated grasping. It should also be noted that "self-adaptive" has already been used by other authors to describe these systems (Rubinger et al., 2001; Carrozza et al., 2004). They are also sometimes referred to as "intelligent" systems (Ulrich, 1988; Gosselin, 2005), which is more vague and therefore, should probably be avoided.

Correspondence to: L. Birglen

(lionel.birglen@polymtl.ca)
The objective of self-adaptive mechanisms is to delegate part of the control tasks from the electronic board to the mechanical layout of the system itself. Hence, with selfadaptive robotic hands, parts of the possible motions of the fingers have to be uncontrolled electronically. However, these motions must be carefully predicted and studied in order to achieve the desired closing sequence, or else, they can lead to degenerate behaviours (Birglen and Gosselin, 2006b). Since some degrees of freedom (DOF) of these hands are not controlled, they are often referred to as "underactuated" (Laliberté and Gosselin, 1998). Again, this adjective is technically correct but has also lead to some confusion. Underactuation in robotic hands is different from the concept of underactuation usually presented in robotic systems and the differences between both notions should be made clear. An underatactuated serial robot is defined as a manipulator with one or more unactuated joints. On the other hand, "underactuated" or self-adaptive fingers use passive elements (the most common of which are springs) in the design of their unactuated joints. Thus, one should rather think of these joints as uncontrollable or passively driven instead of unactuated.

With self-adaptive robotic systems, and conversely to usual underactuated manipulators, the actuation torque (or force) is distributed to each joint of the system. This distribution property is essential and can be related to the Transmission matrix of the linkage (Birglen, 2009) which is characteristic of the type of transmission mechanism required to achieve this distribution. In principle, this distribution 


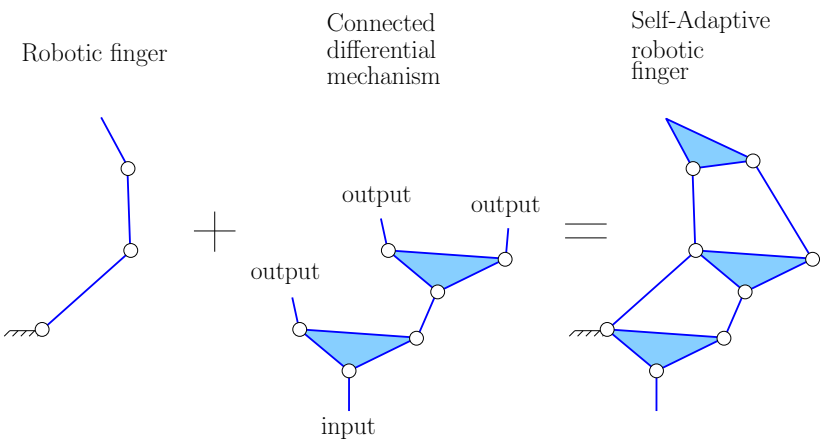

Figure 1. A connected differential mechanism driving a robotic finger.

property is similar to the behaviour of differential mechanisms. Let us recall that according to the IFToMM terminology (IFToMM, 1991), a differential mechanism is a twoDOF mechanism that may resolve a single input into two outputs and vice versa. Therefore, if more than two outputs are required - typically three with robotic fingers (for three phalanges) - the simplest approach is to stack multiple differential devices, each stage adding one DOF to the system (Hirose, 1985; Birglen and Gosselin, 2006c). An illustration of this process is given in Fig. 1, the outputs of two differential seesaw mechanisms (middle, presented in Birglen and Gosselin (2006c)) connected in series drive a robotic finger (left) resulting in a well-known architecture of self-adaptive robotic finger (right), proposed in Gosselin and Laliberté (1996) and consequently used in several prototypes (Laliberté et al., 2002).

However, the designs obtained with this method are only a small sample of a vastly larger number (literally thousands) of possible mechanisms with equal or less complexity (Birglen, 2009). Hence, differential mechanisms is also not the best term to describe self-adaptive mechanisms.

Passive elements are the second ingredient to selfadaptation and are used to kinematically constrain the system. Strictly speaking, the inclusion of a specific passive element is not absolutely necessary to achieve selfadaptation since inertial properties can be used as "passive elements" (Birglen, 2009). In this particular case, the resulting linkage is close to the meaning of underactuation commonly found in the literature. However, to the best of the author's knowledge only two prototypes of self-adaptive fingers have importantly relied on dynamic parameters such as inertia, namely from Higashimori et al. (2005); Crisman et al. (1996). Since the most common passive element by far is the spring (preloaded or not), it has been deemed mandatory. This is not true, compliance is not at all necessary. For instance, other passive elements are presented in Birglen (2009).

As mentioned before, underactuation in grasping has lead to some misconception in the past as to which systems it de-

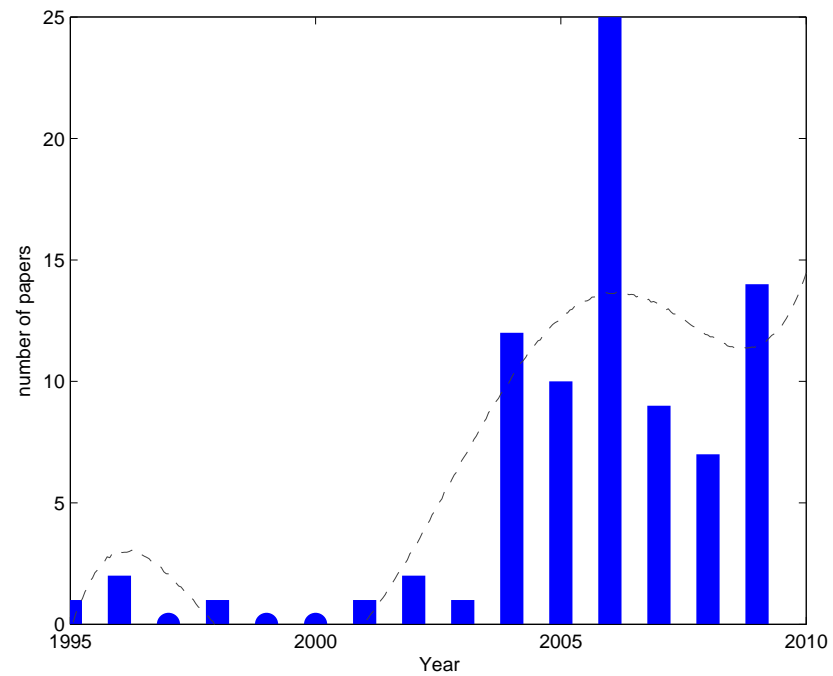

Figure 2. Publication activity in underactuated grasping.

scribed because "underactuation" was associated with underactuated manipulators. This has lead the author to start using "self-adaptive" with an increased frequency. However, this might not be necessary in the coming years as underactuation in grasping is getting more well-known. Indeed, the number of papers published on underactuation in grasping has been steadily increasing in the past few years as illustrated in Fig. 2. The data from this figure have been obtained by searching the following terms on all the available databases from the Engineering Village Database ${ }^{1}$ :

\section{(((underactuated) OR (self-adaptive)) AND ((hand*) OR (finger*) OR (gripper*))) WN TI}

Note that the duplicates and obviously out of scope entries have been manually removed. The total number of references found with this search is 85 . Of course, the results of this search engine are not complete. For instance, well-known prototypes such as the ones discussed by Hirose and Umetani (1978); Townsend (2000); Kyberd et al. (2001) do not appear. Additionally, the author's own database of publications has recently reached 500 entries (excluding patents) on underactuated grasping and related topics. However, this test is representative of the level of activity on this topic and has the advantage of being repeatable by anyone (thus verifiable). The author is pondering the diffusion of his database of publications on a public website similarly to what Prof. Bonev did with the ParalleMIC ${ }^{2}$ for parallel mechanisms. One can see that during the last ten years, the number of publications on underactuated grasping exploded and this area of research is now not anecdotal anymore. Nevertheless, it is also obvious that it is not yet a "mainstream" issue in robotics. It is the author's opinion that we are only at the beginning of the

\footnotetext{
${ }^{1} \mathrm{http}: / / \mathrm{www}$.engineeringvillage2.org

${ }^{2} \mathrm{http}: / /$ www.parallemic.org/
} 


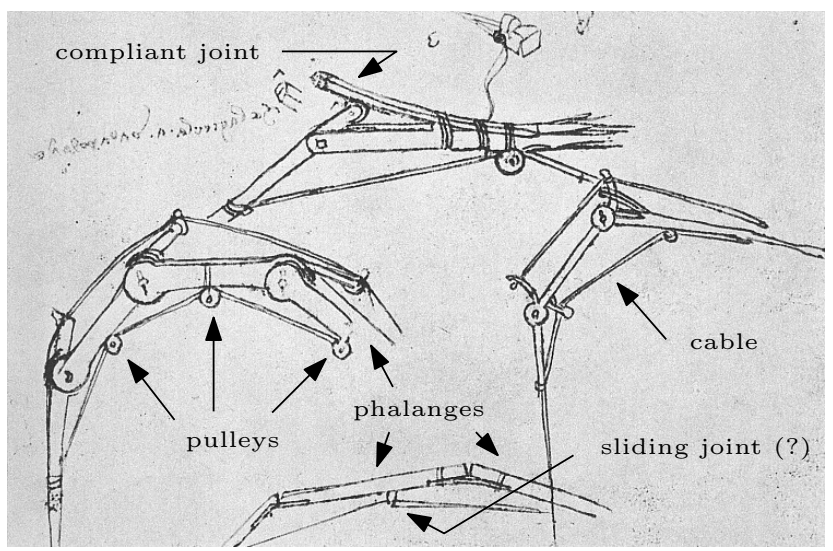

Figure 3. Mechanical wing from Da Vinci's Codex Atlanticus (ca 1496) annotated by the author.

curve illustrated in Fig. 2 and that a new trend in robotics is emerging for underactuation in grasping is only a small sample of the possible applications of self-adaptive mechanisms. As an example to illustrate this claim, let us consider the oldest self-adaptive linkage known to the author: Da Vinci's articulated wing (Birglen et al., 2008).

\section{Da Vinci’s linkage}

\subsection{Introduction}

Leonardo Da Vinci proposed in the Codex Atlanticus a mechanism illustrated in Fig. 3 based on a cable and pulleys that was intended to drive an artificial wing in one of the fantastic flying machine developed by the Renaissance genius.

It is unknown if such a wing or machine has ever been actually tested let alone built by Da Vinci but it proves the ingenuity of engineers before the discovery of electrical actuators. The mechanism itself consist of several sections, similar to phalanges, whose motion was driven by a cable pulled by the human pilot of the machine. This cable was attached to the distal phalanx and ran through the phalanges with the help of pulleys or (maybe) sliding joint. The actual design of this wing bears a striking similarity with an anatomic sketch of the human finger found in another of Da Vinci's codex (see Birglen et al., 2008, for a comparison). More than four hundred years later a very similar design was patented for a robotic finger (Rovetta et al., 1982). The first prototype of modern underactuated fingers, namely the Soft-Gripper of Prof. Hirose (Hirose and Umetani, 1978), was also an architecture in which a cable is run through a series of pulleys until the distal end of the finger. Although in that case, the similarity between the two mechanisms ends here since the actual design of the Soft-Gripper is quite different from Da Vinci's architecture. It is nonetheless interesting to note that this first modern prototype which popularized underactua-

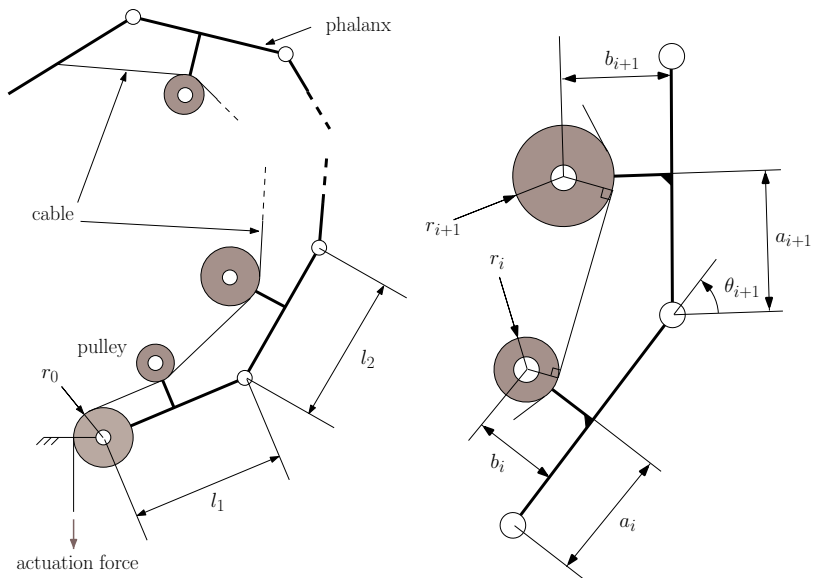

Figure 4. Da Vinci's mechanism.

tion in grasping among the research community shares many traits with Da Vinci's linkage.

\subsection{Grasping}

The architecture discussed in this section is shown in Fig. 4, and corresponds to a general model of the drawing found in the Codex Atlanticus. Notice that all the pulleys of this linkage can freely rotate around their axes.

The Transmission matrix of this mechanism is Birglen et al. (2008):

$\mathbf{T}=\left[\begin{array}{ccccc}1 & \frac{R_{1}}{r_{0}} & \frac{R_{2}}{r_{0}} & \ldots & \frac{R_{n-1}}{r_{0}} \\ \mathbf{0}_{n-1} & & \mathbf{1}_{n-1} & & \end{array}\right]$

where $\mathbf{0}_{n-1}$ and $\mathbf{1}_{n-1}$ are respectively the null vector and identity matrix of dimension $n-1$. The radius of a pulley equivalent to the $i$-th transmission stage (illustrated in the righthand side of Fig. 4 ) is noted $R_{i}$ for $i=1, . ., n-1$. The linkage is driven by a base pulley (with a radius $r_{0}$ ) illustrated in the left-hand side of Fig. 4. It can be shown that the equivalent radius of the $i$-th transmission stage is

$R_{i}=r_{i}+\frac{b_{i}(r b-a l)-\left(l_{i}-a_{i}\right)(a r+b l)}{a^{2}+b^{2}}$

with

$$
\begin{array}{r}
r=r_{i+1}-r_{i}, \\
a=l_{i}-a_{i}+a_{i+1} \cos \theta_{i+1}-b_{i+1} \sin \theta_{i+1}, \\
b=-b_{i}+a_{i+1} \sin \theta_{i+1}+b_{i+1} \cos \theta_{i+1}, \\
l=\sqrt{a^{2}+b^{2}-r^{2}} .
\end{array}
$$

When computing this equivalent radius, one must take into account that the points where the cable comes in contact with the pulleys $r_{i}$ and $r_{i+1}$ is variable and depend on the angle $\theta_{i+1}$. Similarly to the analysis of the Soft Gripper presented in (Birglen et al., 2008), the conditions for the forces to become zero are implicit functions that cannot be easily solved. 
Contact Force F1 (N)

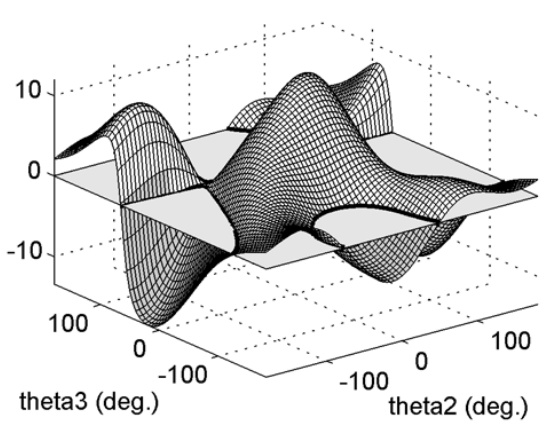

Contact Force F3 (N)

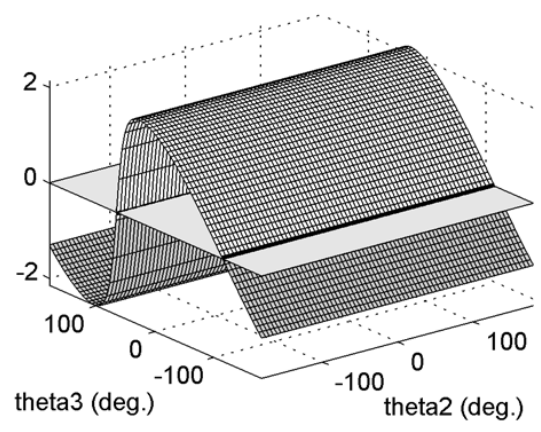

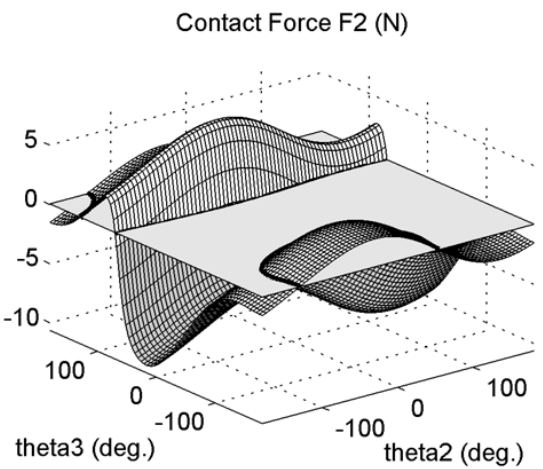

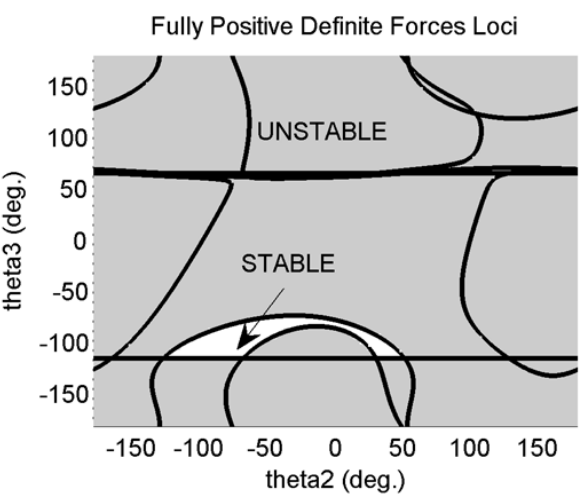

Figure 5. Contact forces and stability loci of Da Vinci's mechanism.

A section of the contact force workspace of the three-phalanx finger based on Da Vinci's drawing is illustrated in Fig. 5 for contact locations at mid-phalanx. The geometric parameters used in this example are directly measured from a copy of the Codex Atlanticus drawings. Note that conversely to the analysis of the same architecture found in (Birglen et al., 2008), the parameters used in this paper are these that are illustrated in the lower part of Fig. 3, i.e. with a zero-radius pulley which models a sliding joint. It seemed to be the preferred design of Da Vinci since he used it in several other sketches of his flying machines.

As can be seen in Fig. 5, the fully positive workspace i.e. the part of it corresponding to the case where all the contact forces are positive or null - of this architecture is rather small. This is especially true when comparing to Prof. Hirose's Soft Gripper. However, as discussed in Birglen and Gosselin (2006b), a fully positive workspace for threephalanx finger might be actually impossible and more importantly, not really desirable. The analysis of the grasp stability of the finger which aims at predicting if the mobility of a self-adaptive finger will converge to a stable equilibrium or towards ejection of the object from the finger is more meaningful. Yet, this analysis is challenging especially with threephalanx fingers (Birglen and Gosselin, 2006a). In this paper, focus is placed on the analysis of a simpler two-phalanx version of the finger, using the grasp-state plane. The geometric parameters associated with this design are listed in Table 1.
Table 1. Da Vinci's two-phalanx mechanism geometric parameters.

\begin{tabular}{ccccccc}
\hline$l_{1}$ & $l_{2}$ & $r_{0}$ & $a_{1}$ & $b_{1}$ & $r_{1}$ & $a_{2}$ \\
\hline 1 & 2.06 & 1.03 & 2.08 & 0 & 0 & .45
\end{tabular}

The grasp-state plane shows if, for an arbitrary initial contact situation, the finger will be stable or not. And, if not, if the subsequent motion undergone by the finger will converge towards a stable equilibrium or ejection. The initial and final grasp-state associated with the finger under scrutiny are illustrated in Figs. 6 and 7. In the initial grasp-state plane, the areas in gray indicate that at least one contact force is negative which means that a full enveloping grasp with both phalanges is impossible. Then, a sliding motion of the finger along the surface of the object will begin and either reach a situation where the finger is in static equilibrium (resulting in a stable pinch grasp) or lose the object (ejection). In Fig. 7, the areas in gray correspond to ejection while the white zones indicate an eventual static equilibrium. As can be seen, the finger is mostly stable for negative angles of the distal phalanx which is not usually the part of the workspace where the finger is used. 


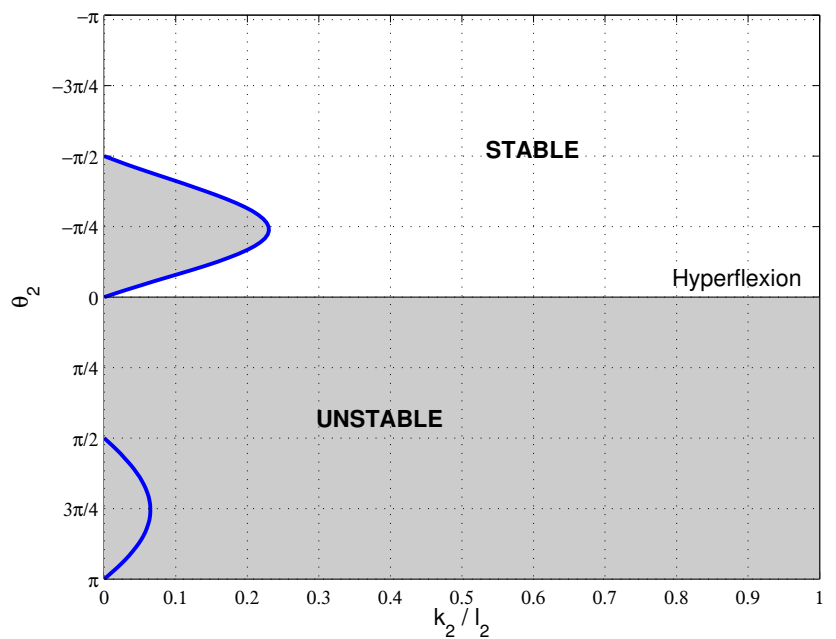

Figure 6. Initial grasp-state plane of Da Vinci's finger.

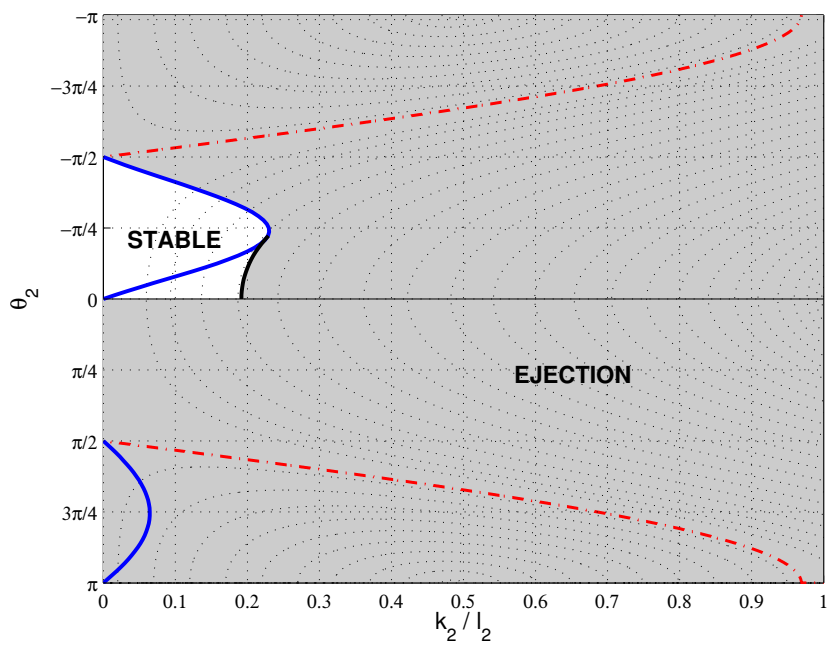

Figure 7. Final grasp-state plane of Da Vinci’s finger.

\subsection{Flying}

From the results of the previous section, we can say that the architecture of Da Vinci makes a poor underactuated finger. Using the same type of transmission, the Soft-Gripper architecture is a far better choice and generally speaking, an excellent gripper. Of course, to be fair, Da Vinci intended his design to be part of a flying machine not a grasping finger so we cannot belittle his invention. For his particular application, Da Vinci also used the shape-adaptation property of the linkage but from an aerodynamic perspective not grasp stability. For a flapping wing, the finger is "in contact" with the air or another fluidic environment. The general motion of a self-adaptive flapping wing constituted of two phalanges is illustrated in Fig. 8. The revolute joint between the two phalanges is equipped with a torsional spring and a mechanical limit. Note that the transmission mechanism achieving this

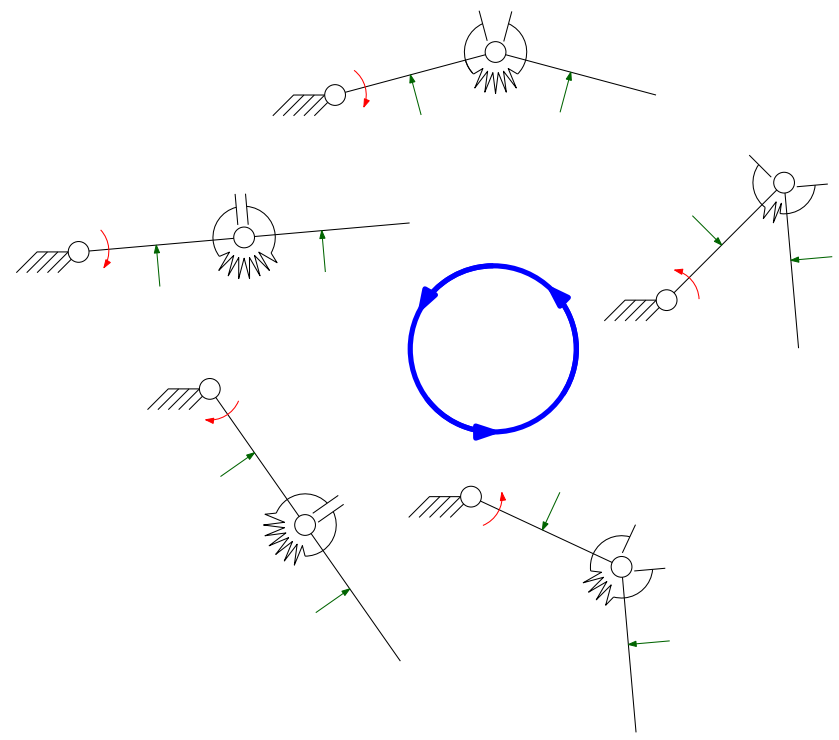

Figure 8. General motion of a self-adaptive flapping wing, the green curved arrows indicate the resistance of the fluid while the red straight arrow symbolize actuation.

motion is not shown for it can take many form, Da Vinci's being one but any other self-adaptive linkage proposed in the literature could be used. If one looks closely to the sketches made by Da Vinci, it is obvious that the human operator of the machine was to provide the actuation force required to the down stroke of the motion while compliant hinges based on cantilever beams were designed to provide the return actuation (cf. Fig. 3). Thus, the idea of using compliant joints in underactuated fingers must be attributed to Da Vinci. This is particularly humbling to note while this idea is recently actively studied by several authors including the one of this paper (Dollar and Howe, 2006; Boudreault and Gosselin, 2006; Doria and Birglen, 2009).

If considering the values of the contact forces was of interest for a grasping finger, in the case of a flapping wing, the torque distributed by the actuator at the base joint of each phalanx might be a more practical quantity. These torques are obviously linked to the contact forces and they are expressed by another form of the Transmission matrix presented in Birglen (2009). However, to establish the upward force developed by this flapping wing, one needs a model for the air/fluid resistance as a function of the shape of the wing and its velocity. This is also necessary to ensure that the motion of the wing will be optimal. The aerodynamic of a flapping wing is a very complex problem and there are only a few non-numerical models available, e.g. in Madangopal et al. (2004). The characterization of the performance of a flapping wing using a self-adaptive linkage is yet an open issue. 


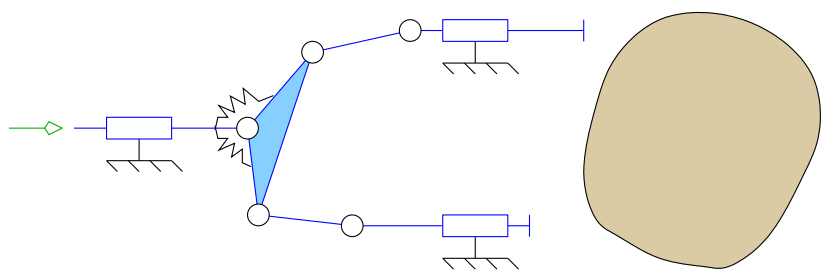

Figure 9. A self-adaptive gripper based on architecture $C 234$.

\section{Other mechanisms and other applications}

The mechanisms synthesized for underactuated grasping do not necessarily have to be used as architectures of robotic fingers or flapping wings using a design based on phalanges. Even for grasping, one can use these mechanisms to synthesize new grippers, as illustrated in Fig. 9. The gripper presented in this figure corresponds to the architecture C234 (Birglen, 2009) and has two-DOF driven by a single actuator. A spring is used as a passive element to constrain the mechanism (it can be used to keep both jaws synchronized during the pregrasping phase), while the leftmost prismatic joint is actuated. A similar mechanism has been proposed to drive two self-adaptive fingers (Birglen and Gosselin, 2006c). Indeed, self-adaptive systems can be connected together to obtain new architectures and this holds for any mechanism properly designed, i.e. not only with differential mechanisms. In the example depicted in Fig. 9, the driven system consists of the two outputs struts with the ground link between them.

Additionally, other designs can be built incrementally from the architectures previously synthesized. Indeed, the known architectures of underactuated fingers are two- or three-DOF mechanisms that can be connected in series or using an arbitrary scheme. Providing that the hypotheses described in Birglen (2009) are satisfied, the resulting architecture will be valid. One can use for instance the motion of the "distal" phalanx of an initial design to drive subsequent phalanges of an extended version of this finger.

Furthermore, the known architectures are not limited to the design of self-adaptive robotic fingers or even grippers but can be extended to a wide range of applications. In Hirose (1985), Prof. Hirose (again) pioneered the design of self-adaptive mechanisms using connected differential mechanisms and proposed to adapt the design he used in the Soft Gripper to a wrist-bracing mechanism for a mobile robot. This robot was to be used to navigate inside a pipe and upon attaining its destination, deploy rigid limbs driven by the selfadaptive mechanism, which allowed them to adapt to the internal shape of the pipe, hence providing stable support to engage in maintenance tasks. With all these systems, the only restriction is that the system reacts to an external constrain of its DOF (i.e. a contact).

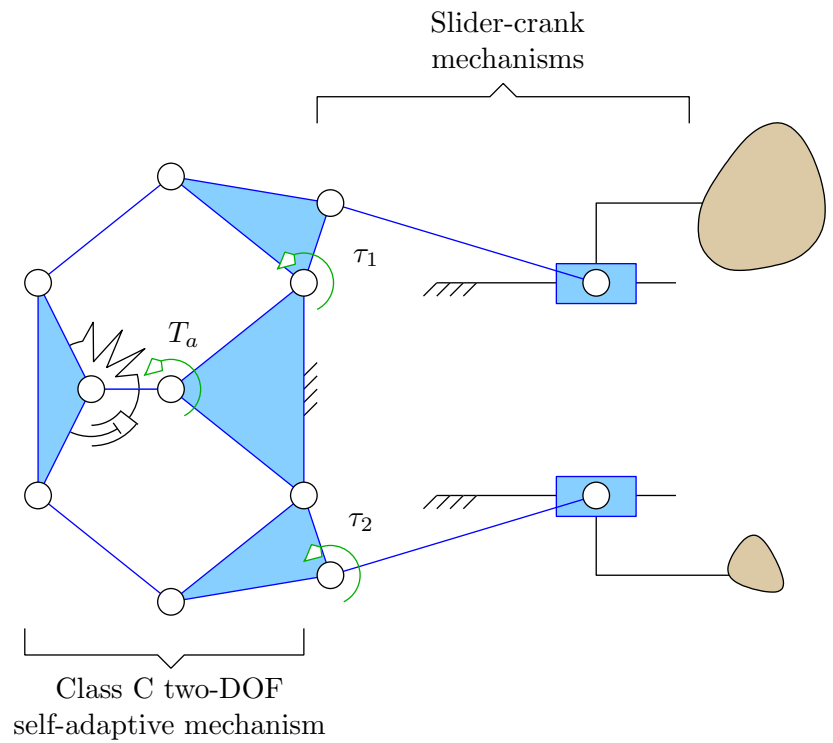

Figure 10. A two-DOF self-adaptive linkage driving two slidercrank linkages.

This restriction itself can be lifted with other types of selfadaptive mechanisms, closely related to the principle of continuously variable transmission. The latter is defined as a technological solution to compensate a variation in the load driven by an actuator axis. Namely, the ability of a mechanical system to continuously change the reduction ratio of its actuator in response to a load variation. These systems are in fact self-adaptive mechanisms too although they have generally only one output, namely the actuator axis. Several mechanisms have been proposed to achieve this capability, mostly based on gears (Hirose et al., 2004; Ishikawa et al., 2000; Fujushima et al., 2001; Takaki and Omata, 2004) (notice that one of these references is from none other than Prof. Hirose again). This principle has yet to be extended to multi-DOF systems but, provided that the actuation distribution condition is satisfied, nothing prevents it. An example of such a mechanism is presented in Fig. 10 where a two-DOF selfadaptive mechanism (the same that was illustrated in Fig. 9) is used to drive two slider-crank linkages. The mechanism is completely symmetrical in order to allow complete revolutions of the joints corresponding to $\tau_{1}$ and $\tau_{2}$. The actuation torque is noted $T_{\mathrm{a}}$. The passive element used here is a spring in parallel with a damper (the choice is arbitrary) which reduces the mechanism into a simple Watt's linkage if $\tau_{1}=\tau_{2}$ (neglecting the dynamics of the linkage itself). If the loads of both slider-crank linkages are different as symbolically illustrated in Fig. 10, the passive element will accommodate this difference by providing another internal mobility. The transmission linkage will still be distributing the actuation torque to both outputs. This accommodation could then be designed to increase the output torque corresponding to the largest load in order to manage the increased load. This is the 
basic principle behind continuously variable transmission as found in the literature. Of course, in this example, it is much simpler to design the system using gears than linkages.

\section{Conclusions}

As discussed in this paper, the topic of underactuated (or selfadaptive) mechanisms recently attracted a lot of interest from the research community. During the Renaissance, Da Vinci designed a flapping wing that has been centuries later used in an underactuated finger. This example was used to illustrate that self-adaptive mechanisms do not have a single application and are therefore currently underexploited. The design of ingenious mechanisms is nowadays mainly a lost art due to the ever progressing of electronics. Yet, this skill can be of tremendous interest in modern robotic and mechatronic devices. The author strongly believes that the research community has to put back more focus on the mecha- in mechatronics if the best performance are to be obtained or if practical success is required. As a well-known senior researcher once said: kinematics' not dead (Merlet, 2000)!

Acknowledgements. The financial support of the Natural Sciences and Engineering Research Council of Canada (NSERC) and the Fonds Québécois de la Recherche sur la Nature et les Technologies (FQRNT) is gratefully acknowledged.

Edited by: J. L. Herder

Reviewed by: three anonymous referees

\section{References}

Birglen, L.: Type Synthesis of Linkage-Driven SelfAdaptive Fingers, J. Mechanisms Robotics 1, 021010 , doi:10.1115/1.3046139, 2009.

Birglen, L. and Gosselin, C.: Geometric Design of Three-Phalanx Underactuated Fingers, ASME Journal of Mechanical Design, 128, 356-364, 2006a.

Birglen, L. and Gosselin, C.: Grasp-State Plane Analysis of TwoPhalanx Underactuated Fingers, Mech. Mach. Theory, 41, 807$822,2006 b$.

Birglen, L. and Gosselin, C.: Force Analysis of Connected Differential Mechanisms: Application to Grasping, Int. J. Robot. Res., 25, 1033-1046, 2006c.

Birglen, L., Laliberté, T., and Gosselin, C.: Underactuated Robotic Hands, Springer, New-York, 2008.

Boudreault, E. and Gosselin, C.: Design of sub-centimetre underactuated compliant grippers, in: 2006 ASME International Design Engineering Technical Conferences, Philadephia, PA, USA, 2006.

Carrozza, M. C., Suppo, C., Sebastiani, F., Massa, B., Vecchi, F., Lazzarini, R., Cutkosky, M. R., and Dario, P.: The SPRING Hand: Development of a Self-Adaptive Prosthesis for Restoring Natural Grasping, Auton. Robot., 16, 125-141, 2004.

Crisman, J. D., Kanojia, C., and Zeid, I.: Graspar: A Flexible, Easily Controllable Robotic Hand, IEEE Robot. Autom. Mag., 3(2), 32-38, 1996.
Dollar, A. M. and Howe, R. D.: A Robust Compliant Grasper via Shape Deposition Manufacturing, IEEE-ASME T. Mech., 11, 154-161, 2006.

Doria, M. and Birglen, L.: Design of an Underactuated Compliant Gripper for Surgery Using Nitinol, Journal of Medical Devices, 3, 011007, doi:10.1115/1.3089249, 2009.

Fujushima, E. F., Nakamoto, H., Damoto, R., and Hirose, S.: Optimal load-sensitive control for mobile robots equipped with continuously variable transmissions, in: Proceedings of the 2001 IEEE International Conference on Robotics and Automation, Vol. 1, Maui, HI, USA, 476-481, 2001.

Gosselin, C.: Mechanically Intelligent Systems: Smart Designs for High-Performance Robotics, in: Proceedings of the 2005 Canadian Congress of Applied Mechanisms, Montreal, Canada, 74 84, 2005.

Gosselin, C.: Adaptive Robotic Mechanical Systems: A Design Paradigm, ASME J. Mech. Design, 128, 192-198, 2006.

Gosselin, C. and Laliberté, T.: Underactuated mechanical finger with return actuation, US Patent No. 5762 390, 1996.

Higashimori, M., Kaneko, M., Namiki, A., and Ishikawa, M.: Design of the 100G Capturing Robot Based on Dynamic Preshaping, Int. J. Robot. Res., 24, 743-753, 2005.

Hirose, S.: Connected Differential Mechanism and its Applications, in: Proceedings of 1985 International Conference on Advanced Robotics, Tokyo, Japan, 319-325, 1985.

Hirose, S. and Umetani, Y.: The Development of Soft Gripper for the Versatile Robot Hand, Mech. Mach. Theory, 13, 351-358, 1978.

Hirose, S., Tibbetts, C., and Hagiwara, T.: Development of Xscrew: a load-sensitive actuator incorporating a variable transmission, in: Proceedings of the 1999 IEEE International Conference on Robotics and Automation, Vol. 1, Detroit, MI, USA, 193-199, 2004.

IFToMM: Terminology for the Theory of Machines and Mechanisms, Mech. Mach. Theory, 26, 435-539, 1991.

Ishikawa, Y., Yu, W., Yokoi, H., and Kakazu, Y.: Development of Robot Hands with an Adjustable Power Transmitting Mechanism, Intelligent Engineering Systems Through Neural Networks, 10, 631-636, 2000.

Kyberd, P. J., Light, C., Chappel, P. H., Nightingale, J. M., Whatley, D., and Evans, M.: The design of an anthropomorphic prosthetic hands: A study of the Southampton hand, Robotica, 19, 593600, 2001.

Laliberté, T. and Gosselin, C.: Simulation and Design of Underactuated Mechanical Hands, Mech. Mach. Theory, 33, 39-57, 1998

Laliberté, T., Birglen, L., and Gosselin, C.: Underactuation in Robotic Grasping Hands, Japanese Journal of Machine Intelligence and Robotic Control, Special Issue on Underactuated Robots, 4, 77-87, 2002.

Madangopal, R., Khan, Z. A., and Agrawal, S. K.: Biologically inspired design of small flapping wing air vehicles using Four bar mechanisms and Quasi steady aerodynamics, J. Mech. Design, 127, 809-816, 2004.

Merlet, J.: Kinematics' not dead!, in: IEEE International Conference on Robotics and Automation, 2000, Proceedings ICRA'00, Vol. 1, 1-6, 2000.

Rovetta, A., Franchetti, I., and Vicentini, P.: Multi-Purpose Mechanical Hand, US Patent No. 4351 553, 1982. 
Rubinger, B., Fulford, P., Gregoris, L., Gosselin, C., and Laliberté, T.: Self-Adapting Robotic Auxiliary Hand (SARAH) for SPDM Operations on the International Space Station, in: Proceedings of the 6th International Symposium on Artificial Intelligence and Robotics and Automation in Space: i-SAIRAS 2001, Saint-Hubert, QC, Canada, 2001.

Takaki, T. and Omata, T.: Load-Sensitive Continuously Variable Transmission for Robots Hands, in: Proceedings of the 2004 IEEE International Conference on Robotics and Automation, New Orleans, LA, USA, 3391-3396, 2004.
Townsend, W.: The BarrettHand grasper - programmably flexible part handling and assembly, Ind. Robot, 27, 181-188, 2000.

Ulrich, N. T.: Grasping with Mechanical Intelligence, Ph.D. thesis, School of Engineering and Applied Sciences, University of Pennsylvania, Philadelphia, Pennsylvania, 1988. 